\title{
A Report of Two Cases of Bacteremia by Leuconostoc lactis in Immunocompromised Patients
}

\section{Yanmei Ge}

Nanjing Tongren Hospital, School of Medicine, Southeast University

Wenli Ji

Nanjing Tongren Hospital, School of Medicine, Southeast University

Song $\mathrm{Hu}$

Nanjing Tongren Hospital, School of Medicine, Southeast University

Huacheng Tong ( $\sim$ gym8902@163.com )

Nanjing Tongren Hospital, School of Medicine, Southeast University https://orcid.org/0000-0002-01527846

\section{Case report}

Keywords: L lactis, 16S rRNA, venous catheterization, case report

Posted Date: April 19th, 2021

DOI: https://doi.org/10.21203/rs.3.rs-135495/v2

License: (c) (i) This work is licensed under a Creative Commons Attribution 4.0 International License. Read Full License 


\section{Abstract}

Background Leuconostoc lactis ( $L$ lactis) are gram positive cocci and generally considered to be environmental organisms. They were widely regarded as non-pathogenic and therefore of limited clinical significance. Molecular identification is one technique to identify bacteria.

Case reports We presented two cases of bacteremia caused by $L$ lactis. They were both advanced age, history of various diseases, intravenous catheter implantation, parenteral nutrition, longer hospital stay and using of multiple antibiotics. The 16S rRNA genes of the isolates of these two patients' blood were sequenced.

Conclusions When immunocompromised patients with risk factors develop bacteremia caused by $L$ lactis, adequate attention and effective treatment measures should be given.

\section{Background}

Leuconostoc lactis ( $L$ lactis) are gram positive cocci, adynamic, facultative anaerobic, catalase-negative, belonging to the Leuconostoc spp. It is generally believed that $L$ lactis were not pathogenic to human. In 1984 , a study confirmed that leuconostoc was a conditional pathogen which could cause human infections at the first time (1). Since then, they were considered potentially pathogen. There were various cases of infections caused by the microorganism such as brain abscess (2), urinary tract infections (3), catheter-associated bloodstream infections (4) and sepsis (5) .

$L$ lactis were not easily identified due to their coccoid morphology in clinical microbiology laboratory. They used to be reported incorrectly as members of the Streptococcus. Due to all clinical isolates of leuconostoc were resistant to vancomycin, it was important to correctly identify the isolated strains and chose appropriate antibiotics.

In this report, we presented two cases of bacteremia by $L$ lactis in immunocompromised patients. The two cases of $L$ lactis were confirmed by molecular identification.

\section{Case Reports}

Case 1 A-79-yr-old man was admitted to hospital due to weakness of both upper limbs. He had history of alzheimer disease, hypertension and type 2 diabetes for many years. One day after admission, the patient 's body temperature rose to $38.5^{\circ} \mathrm{C}$, white bloodcell (WBC) count, $11.8 \times 10^{9} / \mathrm{L}\left(3.5-9.5 \times 10^{9} / \mathrm{L}, 77.97 \%\right.$ segmented neutrophils); C-reactive protein (CRP) level was $9.06 \mathrm{mg} / \mathrm{L}(0-4 \mathrm{mg} / \mathrm{L})$ and chest CT showed fibrosis of the upper right lung with pleural adhesions. These suggested that he might have lung infection. The patient had many diseases with a long medical history. His immunity was low, so the treatment measures were mainly based on symptomatic treatment, while empiric antimicrobial treatment was used. Continuous of empiric antimicrobial treatment with ceftriaxone, meropenem and moxifloxacin. Pseudomonas aeruginosa was isolated from the sputum cultures, antibiotics were changed to 
tigecycline, imipenem and cilastatin combined with fluconazole. However, the patient's fever persisted and the infection had not resolved. He was transferred to intensive care unit (ICU) due to respiratory failure. Deep venous catheters and tracheotomy were used to meet treatment needs. But chest CT showed his lung infection got worse and CRP level was $43.76 \mathrm{mg} / \mathrm{L}(0-4 \mathrm{mg} / \mathrm{L})$. Two samples of blood cultures were positive for bacterial growth, and $L$ lactis was identified using the VITEK 2 (bioMérieux, Marcy I'Etoile, France) system. Imipenem and cilastatin combined with linezolid were used according to blood culture results. After a period of treatment, the patient's infection symptoms were controlled.

Case 2 A-88-yr-old man, with a previous diagnosis of hypertension and lung cancer. This time he was admitted to hospital in ICU for coma after trauma. Brain CT showed bleeding skulls which was the direct cause of his coma. Taking into account the patient's condition, hemostasis and other symptomatic treatment measures were used after admission. Pseudomonas aeruginosa was isolated in the sputum cultures. According to the results of drug sensitivity test, cefepime, imipenem and cilastatin combined with teicoplanin were used to treat infection until cultures from sputum had no isolates. Since the patient had been in a coma, multiple organ failures started after one month in hospital, tracheotomy and deep venous catheters were used to meet treatment needs. The day after the surgery of venous catheterization, the patient presented a serious infection. His body temperature rose to $39^{\circ} \mathrm{C}$, WBC count, $19.2 \times 10^{9} / \mathrm{L}$ (89.06\% segmented neutrophils); CRP level was $63 \mathrm{mg} / \mathrm{L}(0-4 \mathrm{mg} / \mathrm{L})$; procalcitonin level was $1.28 \mathrm{ng} / \mathrm{ml}$ $(<0.5 \mathrm{ng} / \mathrm{ml})$. Blood cultures yielded Leuconostoc spp. The pathogen was gram-positive cocci and was identified as $L$ lactis. Due to $L$ lactis resistant to vancomycin, piperacillin was used instead of teicoplanin. Then, three blood cultures were taken at different times and all yielded $L$ lactis. Bacteremia caused by $L$ lactis existed persistently. The patient died finally three months after confirmation of $L$ lactis infection.

\section{Microbiological identification}

Two additional sets of blood cultures were collected to obtain microbiological diagnosis of the two patients, repectively. Bacterium growing in all cultures were catalase-negative, alpha-hemolytic grampositive cocci. The colonies were white, small and raised on blood agar (Figure 1). The bacteria was identified as

Leuconostoc spp. by VITEK 2 system. The VITEK MS system (bioMérieux, Marcy l'Etoile, France) was used to identified this clinical bacterial isolate as $L$ lactis quickly and reliably. To obtain more reliable phenotypic data and species identification, the 16S rRNA genes of the isolates of these two patient's blood were sequenced. All sequences were analyzed by BLAST (basic local alignment search tool) and ribosomal database project. The sequence showed $99 \%$ similarity to that of $L$ lactis. Antimicrobial susceptibility tests were detected by diffusion disk method. The microorganism was susceptible to levofloxacin, penicillin, piperacillin-tazobactam, cefoperazone-sulbactam, clindamycin, erythromycin, cefepime and imipenem, but resistant to compound trimethoprim, cefoxitin vancomycin and teicoplanin.

\section{Discussion And Conclusions}


Leuconostoc spp. are generally considered to be environmental organisms, which are commonly found on food products and vegetables. They were widely regarded as non-pathogenic and therefore of limited clinical significance (6). With the increase of cases of infections caused by Leuconostoc spp, they are now considered to be opportunistic pathogens as a rare cause of bacteremia in severely immunocompromised hosts (7-8). By studying, we found that decreased immunity, parenteral nutrition, use of certain antibiotics, such as vancomycin and intravenous catheter implantation were considered risk factors for Leuconostoc spp. infection (9-10).

In our report, we presented two cases of bacteremia infection caused by $L$ lactis. Conservative therapy was implemented after they were admitted to hospital. The two patients were extremely ill and did not respond to therapy. The patients' condition had not improved. They had to be ventilated and given central venous catheters to maintain vital signs. There were many similarities between these two patients. They were both advanced age, history of various diseases, intravenous catheter implantation, parenteral nutrition, longer hospital stay and using of multiple antibiotics. Both of them had risk factors for Leuconostoc spp. infection. Bacterial translocation from the skin exposed to venous catheters could be the source of this pathogen. Bacteremia caused by $L$ lactis had not been previously reported in our hospital.

Since Leuconostoc spp. are naturally resistant to vancomycin, we need to consider the bacteremia caused by them and change the antibiotics in time during the treatment of patients with low immunity and intravenous catheter implantation. The correct identification of isolates in laboratory could guide clinicians to choose the appropriate antibiotics. The automated identification system routinely used in many microbiology laboratories is not reliable to distinguish species. We used the VITEK 2 system to identify bacterial species in our laboratory. In case 2 , we misidentified the isolate as $L$ mesenteroides through this system. Then, we confirmed the bacterium were $L$ lactis by sequence analysis of $16 \mathrm{~S}$ rRNA. This method can reliably identify rare bacterial pathogens (11).

It had been reported that the VITEK 2 system could not accurately identify Leuconostoc spp. at the species level. Compared with the partial sequencing of the 16S rRNA gene, the accuracy rate of the VITEK 2 system was only $15 \%$. Through analysis of the 16S rRNA gene sequence, it was found that most of the isolates of $L$ mesenteroides and $L$ pseudomesenteroides identified by the VITEK 2 system were $L$ lactis (12). Therefore, we recommended that laboratory personnel should simultaneously perform molecular methods and automated tests to identify Leuconostoc spp more accurately.

We should be consider the bacteremia caused by $L$ lactis among patients with low immunity and intravenous catheter implantation. Meanwhile, it's important to identify them timely and accurately. The potential clinical significance of them could help clinical diagnosis and treatment.

\section{Abbreviations}

\section{L lactis: Leuconostoc lactis}




\section{Declarations}

\section{Data Availability}

The data used to support the report are included within the article.

\section{Competing interests}

The authors declare that they have no competing interests.

\section{Authors' Contributions}

WLJ and SH carried out the case collection and laboratory detection. YMG and HCT drafted and revised the manuscript. All authors read and approved the final manuscript.

\section{Acknowledgments}

We would like to thank Nanjing Tongren Hospital Affiliated to Medical School of Southeast University for providing the case and Jiangsu Provincial Hospital for laboratory detection by VITEK MS system. The article was funded by Nanjing Tongren Hospital, School of Medicine, Southeast University.

\section{References}

1. Shlaes D M, Marino J, Jacobs M R. Infection caused by vancomycin-resistant Streptococcus sanguis II. Antimicrobial Agents and Chemotherapy, 1984, 25(4): 527 -8区

2. Albanese A, Spanu T, Sali M, et al. Molecular Identification of Leuconostoc mesenteroides as a Cause of Brain Abscess in an Immunocompromised Patient. J Clin Microbiol. 2006, 48(8): 3044-5.

3. Taneja N, Rani P, Emmanuel R, et al. Nosocomial urinary tract infection due to Leuconostoc mesenteroides at a tertiary care Centre in north India. Indian J Med Res. 2005, 122(2):178-9.

4. Cuervo MSI, Cortés LJ, Rodríguez RE, et al. Leuconostoc sp in cancer patients: A descriptive study. Rev Chil Infectol. 2008, 25(3):184-8.

5. ZE Bi, WU Hong and SHI Yi-li. Leuconostoc lactis sepsis in healthy infant: one case report and literature review. Chin J Infect Control. 2017, 16(10):973-5.

6. Facklam R and Elliott JA. Identification, classification, and clinical relevance of catalase-negative, gram-positive cocci, excluding streptococci and enterococci. Clin Microbiol. 1995, Rev. 8: 479-95.

7. Golan Y, Poutsiaka DD, Tozzi S, et al. Daptomycin for line-related Leuconostoc J Antimicrob Chemother. 2001, 47: 364-5.

8. Menegueti MG, Gaspar GG, Laus AM,et al. Bacteremia by Leuconostoc mesenteroides in an immunocompetent patient with chronic Chagas disease: a case report. BMC Infect Dis. 2018 Nov 
3;18(1):547.

9. Montejo M, Grande C, Valdivieso A, et al. Abdominal abscess due to Leuconostoc species in a liver transplant recipient. J Infect, 2000, 41: 197-8.

10. Bou G, Luis Saleta J L, Sa' ez Nieto J A, et al. Nosocomial outbreaks caused by Leuconostoc mesenteroides mesenteroides. Emerg Infect Dis, 2008, 14: 968-71.

11. C A Petti, C R Polage and P Schreckenberger. The role of 16S rRNA gene sequencing in identification of microorganisms misidentified by conventional methods. J Clin Microbiol. 2005, 43: 6123-25.

12. Lee M R, Huang $Y \mathrm{~T}$, Lee $\mathrm{P} \mathrm{I}$, et al. Healthcare-associated bacteraemia caused by Leuconostoc species at a university hospital in Taiwan between 1995 and 2008. J Hosp Infect. 2011, 78: 45-9.

\section{Figures}

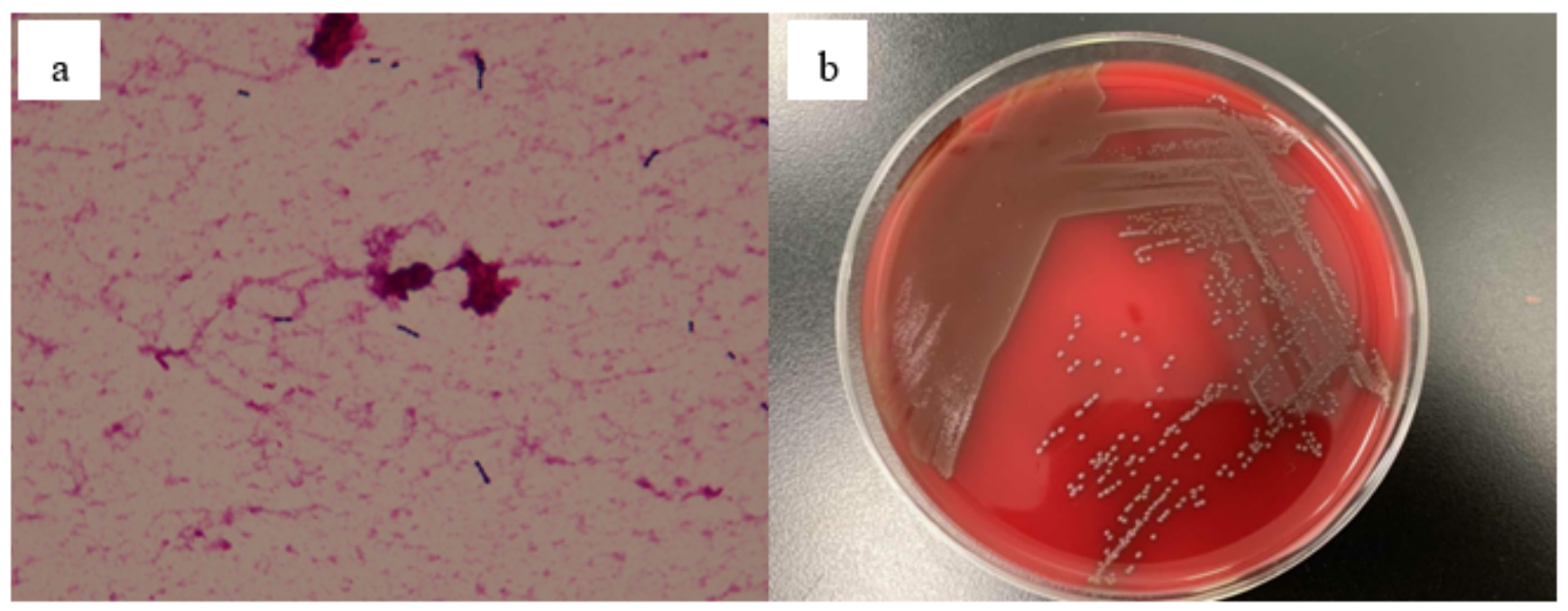

\section{Figure 1}

a. Gram stain showing Gram positive cocci. b. Typical white and small bacteria colonies on blood agar 